Romina Adelina Quevedo-Álava; Luz Adriana Corrales-Moreno; Gema Monserrate Palma-Delgado; Gladis Mercedes Mendoza-Suárez

http://dx.doi.org/10.35381/e.k.v3i5.769

\title{
Psicopedagogía y TIC en período de COVID-19. Una reflexión para el aprendizaje significativo
}

\section{Psychopedagogy and ICT in the COVID-19 period. A Reflection for Meaningful Learning}

\author{
Romina Adelina Quevedo-Álava \\ rquevedo9337@pucem.edu.ec \\ Pontificia Universidad Católica del Ecuador, Portoviejo Manabí \\ Ecuador \\ https://orcid.org/0000-0002-7596-5013 \\ Luz Adriana Corrales-Moreno \\ Pontificia Universidad Católica del Ecuador, Portoviejo Manabí \\ Icorrales3558@pucem.edu.ec \\ Ecuador \\ https://orcid.org/0000-0002-4211-0473 \\ Gema Monserrate Palma-Delgado \\ gpalma3637@pucem.edu.ec \\ Pontificia Universidad Católica del Ecuador, Portoviejo Manabí \\ Ecuador \\ https://orcid.org/0000-0003-3142-7093 \\ Gladis Mercedes Mendoza-Suárez \\ gmendoza7114@pucems.edu.ec \\ Pontificia Universidad Católica del Ecuador, Portoviejo Manabí \\ Ecuador \\ https://orcid.org/0000-0002-7983-2568
}

Recepción: 17 abril 2020

Revisado: 26 de mayo 2020

Aprobación: 17 junio 2020

Publicación: 19 de junio 2020 


\title{
RESUMEN
}

El actual papel de trabajo tiene como objetivo analizar la psicopedagogía y las TIC en período de cuarentena por la pandemia COVID-19 con la finalidad de generar una reflexión para el aprendizaje significativo de los estudiantes del sistema educativo ecuatoriano. Se empleó para ello la descripción documental y análisis de contenido. La psicopedagogía y la educación en general, a la luz de la revisión documental realizada, se encuentra frente al reto de responder efectivamente a las necesidades pedagógicas creadas por la pandemia de COVID-19, esto implica que en relación al abordaje tradicional efectuado en la modalidad presencial, se ubica en una encrucijada sobre su eficacia, por lo que se concibe el inicio de un ciclo de investigación para conocer y adecuar lo educativo a nuevos estilos de aprendizaje, en una etapa social basada en la incertidumbre, resquebrajándose el paradigma educativo de la certeza.

Descriptores: Enseñanza asistida por ordenador; informática educativa; programa informático didáctico; psicología de la educación. (Palabras tomadas del Tesauro UNESCO).

\begin{abstract}
The current work paper aims to analyze psycho-pedagogy and ICT during the COVID-19 period in order to generate reflection for students' meaningful learning in the Ecuadorian educational system. The documentary description and content analysis were used. Psycho-pedagogy and education in general, in light of the documentary review carried out, is facing the challenge of responding effectively to the pedagogical needs created by the COVID-19 pandemic, this implies that in proportion to the traditional approach accomplished in the face-to-face modality, it is located at a crossroads respect to its effectiveness, so the beginning of a research cycle is conceived to know and adapt education to new learning styles, in a social stage based on uncertainty cracking the educational paradigm of certainty.
\end{abstract}

Descriptors: Computer assisted instruction, computer uses in education; educational software; educational psychology. (Words taken from the UNESCO Thesaurus). 
Romina Adelina Quevedo-Álava; Luz Adriana Corrales-Moreno; Gema Monserrate Palma-Delgado; Gladis Mercedes Mendoza-Suárez

\section{INTRODUCCIÓN}

El proceso académico-educativo a causa de la pandemia COVID-19, ha concebido un giro pedagógico en su concepción tradicional de aprendizaje; pues ha sido necesario, para evitar contagios masivos en las escuelas e instituciones educativas, adoptar la modalidad virtual como punto de desarrollo del actual período académico inicialmente pautado, planificado, diseñado, para ejecutarse presencialmente, lo cual obliga a replanificar en función del uso de los diversos recursos en relación a la tecnologías de la comunicación y la información (TIC de aquí en adelante) disponibles en los diversos contextos sociales.

En estas circunstancias, docentes, estudiantes y comunidad educativa en general, confrontan una realidad que a priori se percibe como fácil de manejar; es decir, desarrollar el período académico en modalidad virtual. Sin embargo, al no contarse con la experiencia suficiente en la aplicación de las TIC con fines educativos se genera un problema en el sentido de actuar sobre la marcha para emplear adecuadamente tales recursos digitales. Lo que implica una ruptura en el abordaje educativo que se venía desarrollando en la modalidad presencial; generando a su vez, la necesidad por parte del docente de estudiar las estrategias y herramientas pertinentes para abordar con efectividad el proceso de enseñanza - aprendizaje durante la emergencia por COVID-19. Es allí donde la psicopedagogía como disciplina auxilia de la educación, entra en juego para proponer alternativas configuradas a la realidad actual. Es decir, permite proyectar cómo se debe abordar al estudiante para que éste tenga mayor posibilidad de generar un aprendizaje significativo en consonancia con su realidad social. Al respecto Ortiz \& Mariño (2014), indican que "la psicopedagogía como ciencia con sus problemas, teorías y metodologías propias vinculadas con la formación y desarrollo de educandos y educadores, no solo en contextos escolarizados, sino también en la familia y en la comunidad" (p. 30). 
Romina Adelina Quevedo-Álava; Luz Adriana Corrales-Moreno; Gema Monserrate Palma-Delgado; Gladis Mercedes Mendoza-Suárez

Considerando la anterior definición de psicopedagogía en el momento histórico que se vive, es menester resaltar que la modalidad virtual requiere de atención por parte del docente y del estudiante; además de la inevitable participación de la familia como apoyo inmediato del estudiante, debido a la necesidad de contar con la ayuda para desarrollar aprendizajes mediado por las TIC en un nuevo ambiente como es el hogar, siendo indispensable motivar en función de promover la confianza necesaria en el estudiante para cumplir exitosamente sus compromisos académicos (Hernández, 2018).

Es allí donde el psicopedagogo en conjunto con el docente, deben conocer las diversas realidades sociales de los estudiantes, de ese modo, podrán diseñar estrategias didácticas en función de incentivar un aprendizaje cónsono con la calidad educativa (Messi, Rossi\& Ventura, 2016), siendo ineludible renovar el rol orientador del docente, por cuanto esto contribuye a focalizar las potencialidades de los estudiantes en función de incentivar un aprendizaje en sintonía con la realidad actual (Rojas-Valladares, Estévez-Pichs\& Domínguez-Urdanivia, 2017). Más aun, cuando los críticos pedagógicos cuestionan que por la emergencia transitoria de la enfermedad COVID-19, se tenga que procesar un aprendizaje en línea con fundamento epistemológico que concibe la modalidad de la enseñanza a distancia basada en TIC. En este contexto, Hodges expresa:

Enseñanza remota de emergencia (ERT) es un cambio temporal de la entrega de instrucción a un modo de entrega alternativo debido a circunstancias de crisis. Implica el uso de soluciones de enseñanza totalmente remotas para la instrucción o la educación que de otro modo se impartirían presencialmente o como cursos combinados o híbridos y que volverán a ese formato una vez que la crisis o la emergencia hayan disminuido (Hodges, et al, 2020, p. 3).

Esto representa un indudable reto para los docentes, psicopedagogos, familia, estudiantes, autoridades educativas, por cuanto se requiere generar una reinvención de la educación, basada en fundamentos pedagógicos establecidos de las corrientes en sintonía con el aprendizaje virtual. Sin embargo, al estar frente una emergencia sin antecedentes, podría desarrollarse el ensayo y error para lograr captar el adecuado 
Romina Adelina Quevedo-Álava; Luz Adriana Corrales-Moreno; Gema Monserrate Palma-Delgado; Gladis Mercedes Mendoza-Suárez

abordaje educativo, situación que podría generar estrés, emociones negativas, en los participantes, siendo recurrente involucrar nuevos modos de planificación en donde se incluya el manejo emocional en contexto de la educación por situación de emergencia (Vallejo-Valdivieso, et al, 2019).

Este escenario planteado, aunado a que el Banco Mundial de la Educación (2020), indica que la educación ya se encontraba en crisis antes de la pandemia, hace más desafiante la situación para quienes se encuentran al frente de la responsabilidad de formar a las futuras generaciones, más en un contexto de incertidumbre por no existir la certeza de hasta cuándo durará el aislamiento educativo por COVID-19. Por tanto, los docentes deben recurrir a la innovación educativa como aliada para promover adecuadas herramientas para el fomento del aprendizaje (Jiménez-Galán, 2017). Lo que también implica investigar sobre la marcha de los acontecimientos para aprender haciendo en una sociedad global y dinámica.

Vale resaltar que esto propicia la necesidad de rediseñar la formación de los futuros docentes, así como de quienes se encuentran en ejercicio profesional (Romero \& Villasmil, 2017), considerando lo aprendido en el tiempo de emergencia para desarrollar nuevas estrategias de abordaje educativo, así como para la superación de las debilidades en el aprendizaje. Siendo demandante pare ello, un nuevo modelo organizacional en las instituciones educativas (Eljuri-Blanco \& Villasmil-Yánez, 2018).

En lo que se refiere a las TIC, cabe decir que estas no escapan de la trama planteada, debido a que no estaban consolidadas como modelo educativo antes de la pandemia, sobre todo en los sistemas educativos basados en la presencialidad. Por tanto, entre las TIC, la psicopedagogía, el ejercicio docente, el aprendizaje, se conforma un acumulado de fortalezas y debilidades pedagógicas que deben ser estudiadas durante el desarrollo del período de emergencia, con la finalidad de documentar científicamente, así como pragmáticamente, el aprendizaje desde la práctica, de los aciertos y errores que puedan originarse. A razón de lo planteado, se presenta el actual papel de trabajo enmarcado 
Romina Adelina Quevedo-Álava; Luz Adriana Corrales-Moreno; Gema Monserrate Palma-Delgado; Gladis Mercedes Mendoza-Suárez

en analizar la psicopedagogía y las TIC en período de COVID-19, a fin de generar una reflexión para el aprendizaje significativo de los estudiantes del sistema educativo ecuatoriano.

\section{MÉTODO}

Para el actual artículo de reflexión, se empleó la descripción documental y análisis de contenido, mediante la revisión de escritos relacionados con el tema de investigación, siendo un nivel exploratorio de investigación, por cuanto la dinámica educativa por emergencia causada por el COVID-19, se encuentra en desarrollo, lo que permite abordar antecedentes teóricos en función de construir progresivamente un trabajo investigativo conclusivo, siendo esta la primera fase de trabajo por parte de los investigadores.

\section{ANÁLISIS DE LOS RESULTADOS Y DISCUSIÓN Psicopedagogía y TIC}

La aplicación de las TIC con fines psicopedagógicos se constituye en una alternativa para atender a los estudiantes en el período de emergencia educativa, sin embargo, antes de la pandemia, se hacía recurrente sensibilizar a los docentes y estudiantes sobre el uso efectivo de esta modalidad, lo cual genera la urgencia de hacerlo en el actual tiempo caracterizado por la incertidumbre (Farías-Cedeño, Iñiguez-Auquilla \& Suárez- Romero, 2019).

Lo cual se relaciona con lo planteado por (Romero Oliva \& Montilla-Coronado, 2015), quienes indican que los profesionales de la educación relacionados a la orientación, deben emplear con mayor énfasis las TIC con la finalidad de realizar abordajes psicopedagógicos por esta vía, siendo que son herramientas actuales, sin embargo no existe una cultura de su uso de forma frecuente, lo cual debe subsanarse con un proceso formativo permanente donde se focalice el manejo de los diversos dispositivos, programas, con fines educativos y psicológicos en favor del aprendizaje. 
Romina Adelina Quevedo-Álava; Luz Adriana Corrales-Moreno; Gema Monserrate Palma-Delgado; Gladis Mercedes Mendoza-Suárez

En complemento, (Perochena-González \& Matilde-Coria, 2017), focalizan la atención en generar atención personalizada a los estudiantes, de ese modo, se proyecta en primer orden la construcción de la identidad, es decir, el rol orientador del docente en conjunto con la aplicación psicopedagogas por medio de las TIC, debe envolver el crecimiento personal como factor aliado al aprendizaje, lo cual permite constituir una educación que pone en práctica su esencia, por cuanto no debe perderse de vista tal premisa, más aun, cuando el distanciamiento por el uso de dispositivos tecnológicos, se hace obligatorio.

Lo descrito no puede afectar la socialización propia de la educación, por cuanto las instituciones educativas son un espacio donde los estudiantes crecen en relación al intercambio de valores y comportamientos con sus compañeros, aprenden a cumplir normas, entre otros factores que contribuyen en la formación de una persona en capacidad de convivir son sus semejantes, mediante el cumplimiento de códigos sociales, lo cual no debe estar de lado en la educación virtual por situación de emergencia por COVID-19 (Da-Silva, et al., 2016).

Sin embargo, el abordaje educativo en los hogares como parte de la emergencia por COVID-19, conlleva a plantearse sí el modelo de homeschooling o educación en el hogar (Aliaga-Castillo, 2017), podría ser una alternativa como abordaje pedagógico basado en los intereses y motivaciones de aprendizaje de los estudiantes en conjunto con sus familias, sin embargo esta modalidad ha sido confrontada contra el sistema educativo formal, por lo que podría generarse un espacio conciliador para conformar una interrelación social basada en la generación de valores para la convivencia social.

Así el rol del profesional educativo transciende el contexto habitual, para abordar en las comunidades, hogares, lo relacionado al manejo asertivo de la psicología educativa como un medio para focalizar la interconexión del aprendizaje con la realidad global, caracterizada por la incertidumbre y complejidad del acontecer del devenir social a causa del COVID - 19 (Sánchez-Cabezas, González-Valarezo \& Zumba-Vera, 2016). 
Romina Adelina Quevedo-Álava; Luz Adriana Corrales-Moreno; Gema Monserrate Palma-Delgado; Gladis Mercedes Mendoza-Suárez

La aplicación de las TIC con fines psicopedagógicos se constituye en una alternativa para atender a los estudiantes en el período de emergencia educativa. Sin embargo, antes de la pandemia, se hacía recurrente sensibilizar a los docentes y estudiantes sobre el uso efectivo de esta modalidad, por lo que se genera la urgencia de hacerlo en el actual tiempo caracterizado por la incertidumbre (Farías-Cedeño, Iñiguez-Auquilla \& SuárezRomero, 2019).

En este contexto, Romero Oliva \& Montilla-Coronado (2015), indican que los profesionales de la educación relacionados a la orientación, deben emplear con mayor énfasis las TIC con la finalidad de realizar abordajes psicopedagógicos por esta vía, dado que son herramientas actuales. Sin embargo, no existe una cultura de su uso de forma frecuente, lo cual debe subsanarse con un proceso formativo permanente donde se focalice el manejo de los diversos dispositivos y programas, con fines psicoeducativos en favor del aprendizaje.

A propósito de este abordaje psicopedagógico con énfasis las TIC, Perochena-González \& Matilde-Coria (2017), se focalizan en la necesidad de generar atención personalizada a los estudiantes de ese modo, proyectándose en primer orden la construcción de la identidad. Es decir, el rol orientador del docente en conjunto con la aplicación psicopedagogas por medio de las TIC, debe envolver el crecimiento personal como factor aliado al aprendizaje, lo que permite constituir una educación que pone en práctica su esencia. Por cuanto no debe perderse de vista tal premisa, más aun, cuando el distanciamiento por el uso de dispositivos tecnológicos, se hace obligatorio.

Lo planteado no puede afectar la socialización propia de la educación, por cuanto las instituciones educativas son un espacio donde los estudiantes crecen en relación al intercambio de valores y comportamientos con sus compañeros, aprenden a cumplir normas, entre otros factores. Pues esto contribuye con la formación de una persona capaz de convivir con sus semejantes, mediante el cumplimiento de códigos sociales; por tanto, no debe estar de lado en la educación virtual por situación de emergencia por 
Romina Adelina Quevedo-Álava; Luz Adriana Corrales-Moreno; Gema Monserrate Palma-Delgado; Gladis Mercedes Mendoza-Suárez

COVID-19 (Da-Silva, et al., 2016).

En otro orden de ideas, el abordaje educativo en los hogares como parte de la emergencia por COVID-19, conlleva a plantearse sí el modelo de homeschooling o educación en el hogar (Aliaga-Castillo, 2017), podría ser una alternativa pedagógica basado en los intereses y motivaciones de aprendizaje de los estudiantes en conjunto con sus familias; a pesar de que esta modalidad ha sido confrontada contra el sistema educativo formal, por lo que podría generarse un espacio conciliador para conformar una interrelación social basada en la generación de valores para la convivencia social.

Así el rol del profesional educador transciende el contexto habitual, para abordar en las comunidades y hogares, lo relacionado al manejo asertivo de la psicología educativa como un medio para focalizar la interconexión del aprendizaje con la realidad global, caracterizada por la incertidumbre y complejidad del acontecer del devenir social a causa del COVID - 19 (Sánchez-Cabezas, González-Valarezo \& Zumba-Vera, 2016).

\section{TIC y Aprendizaje significativo}

Las TIC como eje transversal del aprendizaje (Nájar-Sánchez, 2016), contribuye a promover el empoderamiento significativo del conocimiento, por cuanto se basa en la búsqueda y procesamiento de información, situación que le permite al estudiante aproximarse al enfoque centrado en la investigación, como perspectiva de aprendizaje que produce cambios en las estructuras mentales, a razón de que la información se fija para ser procesada a propósito de resolver problemas y aplicarla en la vida cotidiana (Briceño-Ruperti, Vallejo-Valdivieso \& Moya-Martínez, 2019).

Desde este enfoque, que se centraliza en el estudiante, las TIC promueven su participación activa con la finalidad de propiciar la construcción del conocimiento (BurgosBriones, Pico-Barreiro \& Vélez-Zambrano, 2019). Por tanto, es una alternativa necesaria en medio de la emergencia por COVID-19, pues se requiere el despliegue de experiencias donde el estudiante desarrolle un aprendizaje, no solo en conformidad de 
Romina Adelina Quevedo-Álava; Luz Adriana Corrales-Moreno; Gema Monserrate Palma-Delgado; Gladis Mercedes Mendoza-Suárez

cumplir con las asignaciones académicas; sino también, que se interconecte con la realidad social. Pues de esta manera se proseguirá con el rol educativo de formar, superando el modelo mecanicista; aunque se corra el riesgo que este se encuentre presente si no se produce realmente un cambio de modelo al implementar las TIC.

Es importante saber que una posibilidad de fomentar el aprendizaje significativo, es mediante la realización de proyectos productivos por medio de las TIC (Lattá-Arias, 2019). De este modo el estudiante tiene la oportunidad de poner en práctica las diversas competencias que posee; además que le permite descubrir nuevas habilidades, aunado al trabajo cooperativo y colaborativo con sus compañeros desde ambientes virtuales. Por consiguiente, articular un modelo educativo desde esta perspectiva que pueda cumplir con las necesidades académicas y resolver situaciones sociales en medio de la emergencia por COVID-19, permitiría a los psicopedagogos y docentes en su rol orientador, conocer las fortalezas y debilidades de los estudiantes, con la finalidad de diseñar planes de trabajo que contribuyan a superar los inconvenientes en razón de acceder a una educación inclusiva y de calidad.

En todo este contexto del aprendizaje enfocado en el estudiante y centrado en la investigación y los proyectos productivos mediados por las TIC, la integración de saberes un indicador de que este ha logrado un aprendizaje significativo (Jaramillo-Naranjo, 2019). Pues se genera la configuración neuronal que produce capacidades cognitivas para la construcción de conocimientos articuladores de soluciones a diversas problemáticas. Siendo necesaria para tal fin, la autorregulación por parte del estudiante en razón de trabajar a partir de las emociones en concordancia con potenciar sus capacidades metacognitivas por medio de la auto motivación (Martínez Albán, VallejoValdivieso \& Moya-Martínez, 2019). 
Romina Adelina Quevedo-Álava; Luz Adriana Corrales-Moreno; Gema Monserrate Palma-Delgado; Gladis Mercedes Mendoza-Suárez

\section{Psicopedagogía y TIC en emergencia por COVID-19}

En este en período de confinamiento por COVID-19, parte de la psicopedagogía no solo se debe limitar a gestionar procesos acordes para la generación de aprendizaje en la modalidad virtual, asumida como un enfoque de emergencia sustituto de la presencialidad. También, debe considerar la situación de los estudiantes que no poseen las condiciones sociológicas como conectividad a internet, equipos tecnológicos, aunado a quienes poseen algún tipo de discapacidad que minimiza o impide su participación de un modo activo. En este sentido, Vohlonen (2020), plantea:

En Ecuador, solo el 37 por ciento de los hogares tiene acceso a internet, lo que significa que 6 de cada 10 niños no pueden continuar sus estudios a través de plataformas digitales. La situación es más grave para los niños de zonas rurales, solo el 16 por ciento de los hogares tiene este servicio. No obstante, en el país 9 de cada 10 hogares sí cuenta con televisor o teléfono celular, lo que supone una alternativa para llegar a los niños a través de estos medios (p. 1).

Situación que amerita la debida atención por cuanto rompe con el idealismo de lograr una educación uniforme y en igualdad de condiciones, cuando se trata de abordar la emergencia educativa causada por COVID-19, mediante la modalidad virtual. Causa similar es planteada por (Fernández-Enguita, 2020), al indicar que existen brechas que originan distanciamiento entre los estudiantes españoles, entre las cuales se encuentran:

1. Brecha de acceso (tener o no tener acceso a conexión y dispositivos tecnológicos). La distribución es muy desigual según el nivel socioeconómico de los alumnos: en el nivel bajo, el $14 \%$ de los alumnos no tienen ordenador en casa, mientras que un $44 \%$ solo tiene uno. Estas cifras contrastan con las del grupo socioeconómico alto, donde el $61 \%$ dispone de tres o más ordenadores en casa, el $31 \%$ dispone de dos, y únicamente un $8 \%$ tiene tan solo uno.

2. Brecha de uso (tiempo de uso y calidad del mismo). Los alumnos españoles se encuentran en niveles similares de uso con respecto a otros países. $Y$ aunque apenas se muestran diferencias por comunidades autónomas, sí se encuentran 
Romina Adelina Quevedo-Álava; Luz Adriana Corrales-Moreno; Gema Monserrate Palma-Delgado; Gladis Mercedes Mendoza-Suárez

por nivel socioeconómico con respecto al uso de dispositivos TIC en el hogar

3. Brecha escolar (habilidades del profesorado, disponibilidad de recursos y adecuación de plataformas online de apoyo a la enseñanza). De acuerdo con los equipos directivos, aproximadamente un $50 \%$ de ellos afirma que sus docentes disponen de capacidades y recursos profesionales para aprender a integrar los dispositivos digitales en la enseñanza; igual porcentaje que el de alumnos que se encuentran en centros que cuentan con una plataforma online eficaz.

Lo planteado es un indicador de que las sociedades, sin tener en cuenta su ubicación continental, en cierto modo no están del todo preparadas para asumir con efectividad y pertinencia el enfoque virtual. Esto, no solo obliga a repensar el funcionamiento de las instituciones educativas; sino también, el contexto social basado en la economía como escenario central que no puede estar relegado de la educación. Pues el momento histórico da para reflexionar sobre el acontecer educativo de cara a los próximos tiempos marcados por la incertidumbre, lo cual avizora que el tiempo de la seguridad del paradigma racionalista podría estar desplazada por un nuevo modelo en continua construcción. 
Romina Adelina Quevedo-Álava; Luz Adriana Corrales-Moreno; Gema Monserrate Palma-Delgado; Gladis Mercedes Mendoza-Suárez

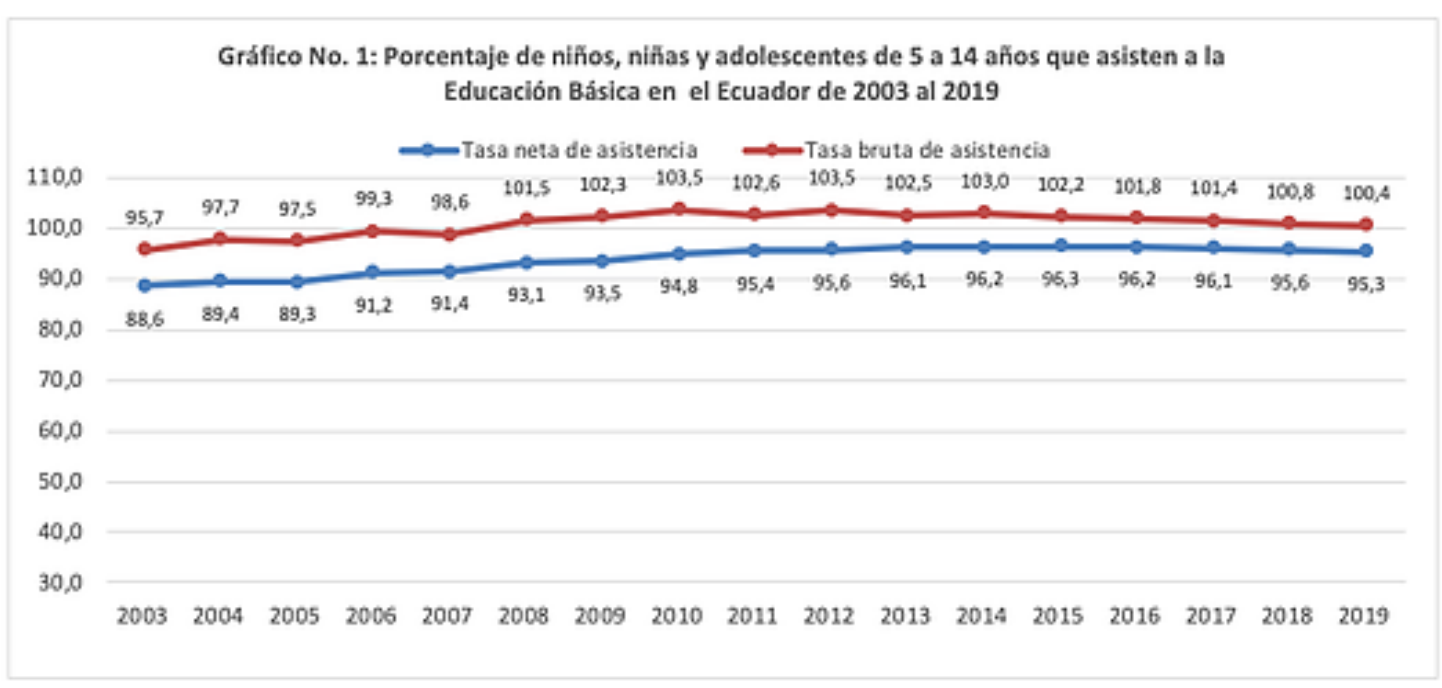

Figura 1. Participación de estudiantes en el Ecuador 2013-2019.

Fuente: Observatorio Social del Ecuador (OSE, 2020).

En otro orden de ideas y considerando la figura 1 presentada, se evidencia que la participación de los estudiantes en el Ecuador en la modalidad presencial ha sido hasta del $96 \%$. Esta estadística se puede tomar como referencia para comparar con cifras que, aunque aún no se cuentan, en próximas investigaciones permitiría establecer el nivel de deserción en el 2020, a modo de confirmar que la educación ecuatoriana no sido fundamentalmente generada desde la virtualidad. Lo cual repercute en el ámbito de la psicopedagogía en estudiar las consecuencias que podrían generarse a partir de la emergencia por COVID-19.

En lo que respecta a la innovación educativa, es necesario la creación de laboratorios en donde se diseñen recursos virtuales para el aprendizaje en correspondencia a la psicopedagogía. Esto permitiría evitar la improvisación o el ensayo - error que podría originarse al no estar habituados pedagógicamente al uso de las TIC, con un modelo centrado en la indagación y resolución de problemas, que promueve competencias en el estudiante. Sin embargo, con la visión de emergencia actual, se corre el riesgo de implementarlo como una panacea sin desarrollar efectivamente los principios filosóficos 
Romina Adelina Quevedo-Álava; Luz Adriana Corrales-Moreno; Gema Monserrate Palma-Delgado; Gladis Mercedes Mendoza-Suárez

del enfoque basado en aprendizaje mediado por las TIC.

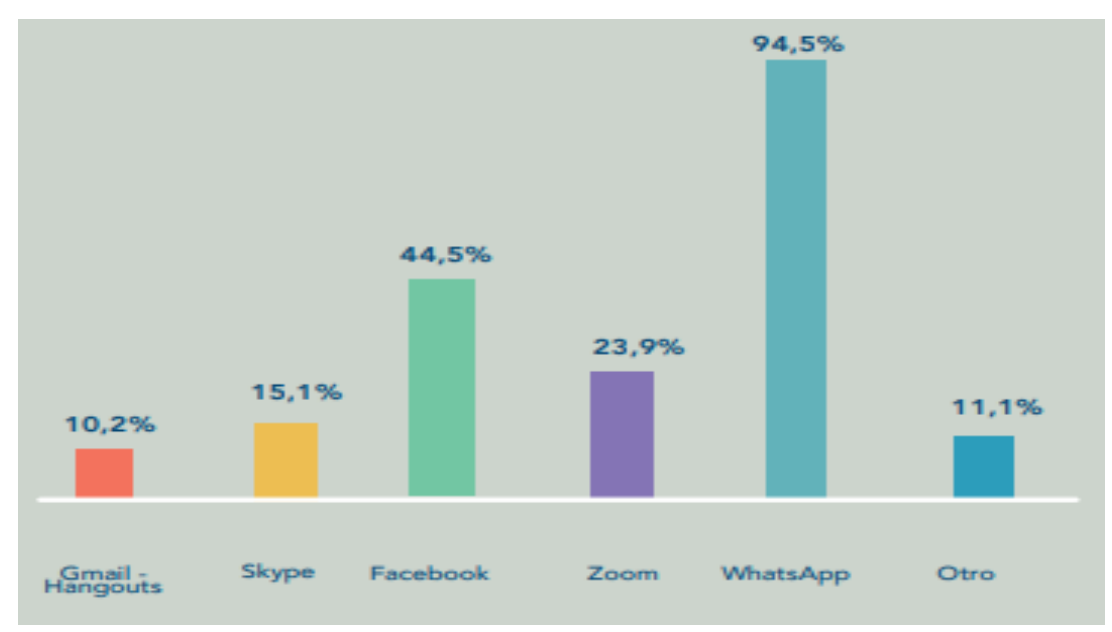

Figura 2. Plataformas más usadas en Ecuador durante período de emergencia.

Fuente: Grupo Faro, 2020.

Con la figura 2 se muestra cual ha sido la plataforma más usada en Ecuador durante período de emergencia. En este sentido, el uso mayoritario de whatsaap, evidencia que es el recurso más accesible y manejable por el entorno del estudiante. Esto repercute en la necesidad de diseñar estrategias psicopedagógicas en favor de estimular el aprendizaje significativo, siendo necesaria una actuación multidisciplinar para lograr tal fin, aunado a prestar atención al nivel socio económico, así como la formación para emplear otras plataformas o recursos para gestionar la posibilidad de construir conocimientos complejos.

También se hace necesario emplear métodos alternativos como la realidad virtual, con la intención de mejorar la calidad educativa (Cantón-Enríquez, et al, 2017). En este sentido es menester invertir para el establecimiento de entornos virtuales de aprendizaje basados en esta metodología, lo cual permite al estudiante aprender en forma significativa desde ámbitos 
Romina Adelina Quevedo-Álava; Luz Adriana Corrales-Moreno; Gema Monserrate Palma-Delgado; Gladis Mercedes Mendoza-Suárez

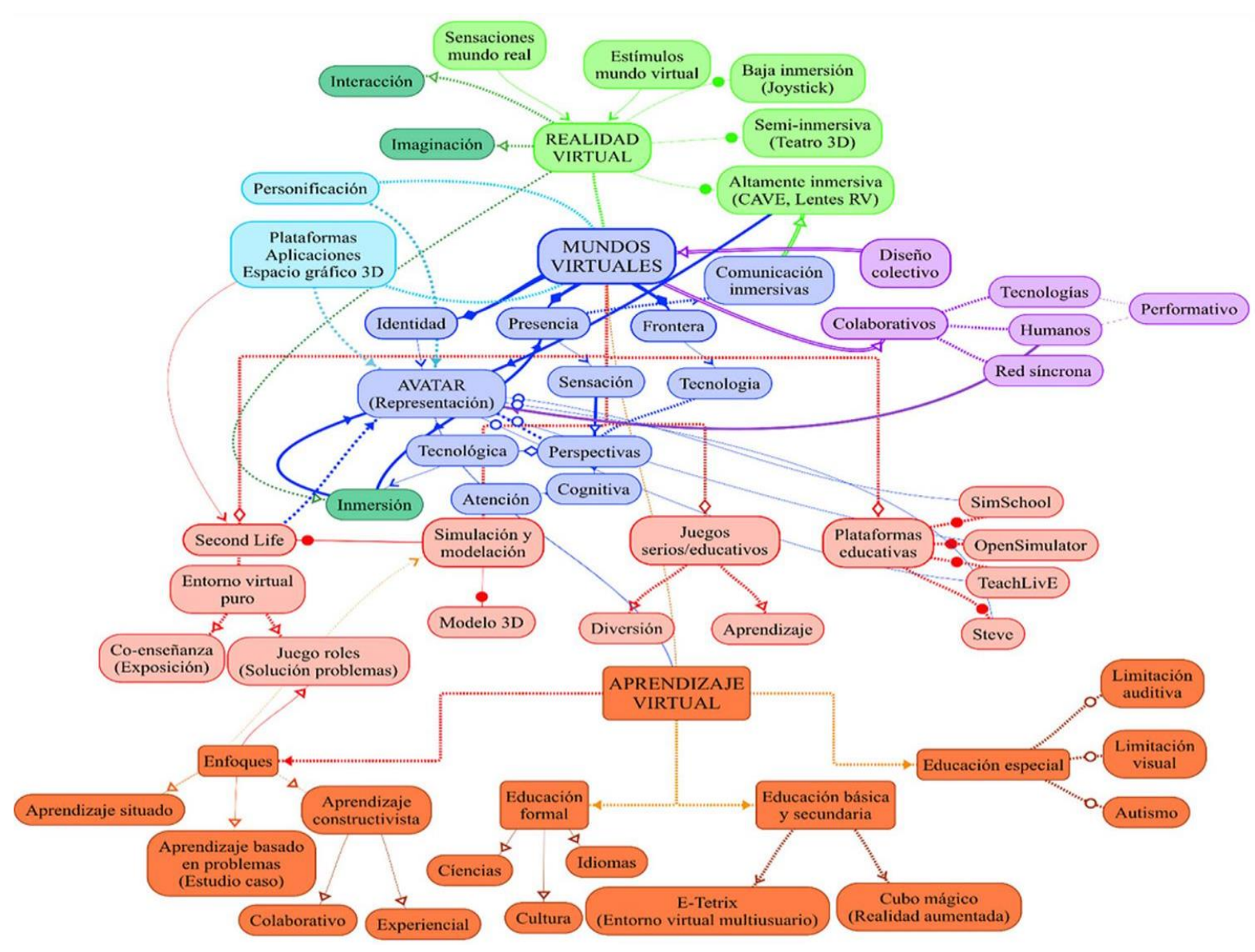

Figura 3. Entornos virtuales de aprendizaje en realidad aumentada. Fuente: Toca-Torres \& Carrillo-Rodríguez (2019).

Las posibilidades de implementar diversas opciones de entornos virtuales a la luz de la realidad virtual, facilita que los docentes conozcan de programación, puedan diseñar sus propios recursos y ambientes de aprendizaje contextualizados a las necesidades de la audiencia (estudiantes). Del mismo modo, hace que los estudiantes contribuyan en la construcción de estos recursos, aprendiendo programación y otras competencias informáticas que motiven a aprender temas o contenidos y a involucrar múltiples procesos lógicos - matemáticos para su desarrollo metacognitivo. Además, hace posible que los psicopedagogos y docentes trabajen cooperativamente en el diseño e implementación 
Romina Adelina Quevedo-Álava; Luz Adriana Corrales-Moreno; Gema Monserrate Palma-Delgado; Gladis Mercedes Mendoza-Suárez

de estrategias para el fomento de la metacognición (Mato-Vázquez, Espiñeira\& LópezChao, 2017).

En este tenor, Valenzuela (2019), destaca la importancia de implementar estrategias metacognitivas; sin embargo, considera que aún falta confirmar su efecto en procesos mentales como la divagación y la creatividad. Siendo aquí el ámbito donde la psicopedagogía encuentra una posible línea de investigación e intervención, con la finalidad de responder los vacíos cognitivos evidenciados hasta ahora, sumado a la necesidad de descubrir el comportamiento ante el aprendizaje durante el estado de emergencia por COVID-19. así como propiciar mediante la lectura, una visión reflexiva en los estudiantes como posibilitador de pensamiento sistémico de abordaje de la realidad social (Medina-Coronado \& Nagamine-Miyashiro, 2019).

\section{CONCLUSIÓN}

La psicopedagogía y la educación en general, a la luz de la revisión documental realizada, se encuentra frente al reto de responder efectivamente a las necesidades pedagógicas creadas por la pandemia de COVID-19. Esto implica que en proporción al abordaje tradicional de la enseñanza y el aprendizaje que se efectúa en la modalidad presencial, se ubica en una encrucijada sobre su eficacia.

De allí que se conciba la necesidad de iniciar un ciclo de investigación donde se aborden las experiencias de los docentes con la finalidad de conocer el devenir de la psicopedagogía en la emergencia sanitaria. Esto a fin de adecuar lo educativo a nuevos estilos de aprendizaje, en una realidad basada en la incertidumbre, y donde se resquebraja el paradigma educativo de la certeza. Aunado a transcender las brechas de desigualdad al acceso de internet y recursos pedagógicos virtuales.

\section{FINANCIAMIENTO}

No monetario 
Romina Adelina Quevedo-Álava; Luz Adriana Corrales-Moreno; Gema Monserrate Palma-Delgado; Gladis Mercedes Mendoza-Suárez

\title{
AGRADECIMIENTOS
}

\begin{abstract}
A la Pontificia Universidad Católica del Ecuador, Extensión Manabí, Portoviejo y al Doctor Patricio Alfredo Vallejo-Valdivieso por la orientación ontoepistémica para la construcción de este artículo.
\end{abstract}

\section{REFERENCIAS}

Aliaga-Castillo, L. (2017). Educación en el hogar en Chile: informe de resultados de la Encuesta Nacional. [Home education in Chile: report of the results of the National Survey]. Educación, 26(50), 7-27. Recuperado de https://url2.cl/h3q9E

Banco Mundial de la Educación (2020). Banco Mundial de Educación y COVID-19. [World Bank for Education and COVID-19]. Recuperado de https://url2.cl/BsqVi

Burgos-Briones, J., Pico-Barreiro, L., \& Vélez-Zambrano, G. (2019). El maestro y la educación sostenible 2030. [The teacher and sustainable education 2030]. CIENCIAMATRIA, 6(10), 609-624. https://doi.org/10.35381/cm.v6i10.241

Briceño-Ruperti, M., Vallejo-Valdivieso, P., \& Moya-Martínez, M. (2019). Estructuras mentales y competencia mediática en el aprendizaje significativo. [Mental structures and media competence in meaningful learning]. CIENCIAMATRIA, 5(9), 680-695. https://doi.org/10.35381/cm.v5i9.258

Cantón-Enríquez, D., Arellano-Pimentel, J., Hernández-López, M., \& Nieva-García, O. (2017). Uso didáctico de la realidad virtual inmersiva con interacción natural de usuario enfocada a la inspección de aerogeneradores. [Didactic use of immersive virtual reality with natural user interaction focused on the inspection of wind turbines]. Apertura (Guadalajara, Jal.), $\quad 9(2), \quad 8$ 23. https://dx.doi.org/10.32870/ap.v9n2.1049

Da-Silva C., Fernanda H., Mendes-de-Barros, A., Tono-de-Oliveira, R, De-Andrade, S, Lorenzini-Erdmann, A., \& Dos-Santos, J. (2016). Enfoques teóricos del proceso de socialización en la educación de los enfermeros. [Theoretical approaches of the socialization process in the education of nurses]. Index de Enfermería, 25(4), 258262. Recuperado de https://url2.cl//bnVN 
Romina Adelina Quevedo-Álava; Luz Adriana Corrales-Moreno; Gema Monserrate Palma-Delgado; Gladis Mercedes Mendoza-Suárez

Eljuri-Blanco, A., \& Villasmil-Yánez, M. (2018). Cultura organizacional en docentes de educación básica. [Organizational culture in basic education teachers]. EPISTEME KOINONIA, 1(2), 4-17. http://dx.doi.org/10.35381/e.k.v1i2.508

Farías-Cedeño, J., Iñiguez-Auquilla, B, \& Suárez- Romero, M. (2019). Aplicación de las Tic's en Psicopedagogía. [Application of Tic's in Psychopedagogy]. Revista Espacios. 40(21), p. 9. Recuperado de https://url2.cl/thZjD

Fernández-Enguita, M. (2020). Una pandemia imprevisible ha traído la brecha previsible. [An unpredictable pandemic has brought the predictable gap]. Cuaderno de Campo (31 marzo). Recuperado de https://url2.cl/msU2j

Grupo Faro (2020). Educación en tiempos del COVID-19 en Ecuador. [Education in times of COVID-19 in Ecuador]. Recuperado de https://url2.cl/VXyRf

Hernández, A. (2018). Transitando por el camino de la escuela para padres, madres y representantes. Una experiencia vivida. [Walking on the way to school for parents and representatives. A lived experience]. EPISTEME KOINONIA, 1(1), 51-71. http://dx.doi.org/10.35381/e.k.v1i1.490

Hodges, Ch., Moore, S., Lockee, B., Trust, T. \& Bond, A. (2020). The Difference Between Emergency Remote Teaching and Online Learning. [La diferencia entre la enseñanza remota de emergencia y el aprendizaje en línea]. EducaseReview. Recuperado de https://url2.cl/C8NZK

Jaramillo-Naranjo, L. (2019). Las ciencias naturales como un saber integrador. [Natural sciences as an integrating knowledge]. Sophia, Colección de Filosofía de la Educación, (26), 199-221. https://dx.doi.org/10.17163/soph.n26.2019.06

Jiménez-Galán, Y. (2017). Innovación educativa y docencia ¿falla el protagonista?: el caso ESCOM. [Educational innovation and teaching: does the protagonist fail ?: the ESCOM case]. RIDE. Revista Iberoamericana para la Investigación y el Desarrollo Educativo, 8(15), 710-734. https://dx.doi.org/10.23913/ride.v8i15.317

Lattá-Arias, C. (2019). Uso de las TIC para proyectos productivos en las instituciones educativas del Municipio Zona Bananera. Magdalena. Colombia. [Use of ICT for productive projects in educational institutions of the Municipality of the Banana Zone. Cupcake Colombia]. Revista Arbitrada Interdisciplinaria Koinonía, 4(7), 233246. http://dx.doi.org/10.35381/r.k.v4i7.202 
Romina Adelina Quevedo-Álava; Luz Adriana Corrales-Moreno; Gema Monserrate Palma-Delgado; Gladis Mercedes Mendoza-Suárez

Martínez Albán, W., Vallejo Valdivieso, P., \& Moya Martínez, M. (2019). Estructuras mentales y aprendizaje autorregulado en generación de aprendizaje significativo. [Mental structures and self-regulated learning in generating meaningful learning]. CIENCIAMATRIA, 6(10), 629-645. https://doi.org/10.35381/cm.v6i10.259

Mato-Vázquez, D., Espiñeira, E., \& López-Chao, V. (2017). Impacto del uso de estrategias metacognitivas en la enseñanza de las matemáticas. [Impact of the use of metacognitive strategies in the teaching of mathematics]. Perfiles educativos, 39(158), 91-111. Recuperado de https://url2.cl/3C7Ks

Medina-Coronado, D., \& Nagamine-Miyashiro, M. (2019). Estrategias de aprendizaje autónomo en la comprensión lectora de estudiantes de secundaria. [Autonomous learning strategies in reading comprehension of high school students]. Propósitos y Representaciones, 7(2), 134-146. https://dx.doi.org/10.20511/pyr2019.v7n2.276

Messi, L, Rossi, B, \& Ventura, A. (2016). La psicopedagogía en el ámbito escolar: ¿Qué y cómo representan los docentes la intervención psicopedagógica? [Psychopedagogy in the school environment: What and how do teachers represent psychopedagogical intervention?]. Perspectiva Educacional, Formación de Profesores, 55 (2), 110-128. Recuperado de https://url2.cl/sfrnU

Nájar-Sánchez, O. (2016). Tecnologías de la información y la comunicación aplicadas a la educación. [Information and communication technologies applied to education]. Praxis \& Saber, 7(14), 9-16. https://dx.doi.org/10.19053/22160159.5215

Observatorio Social del Ecuador (OSE, 2020). ¿Estaba el sistema educativo del Ecuador preparado para enfrentar la pandemia de covid-19? [Was Ecuador's education system ready to face the covid-19 pandemic?]. Recuperado de https://url2.cl/2vsnW

Ortiz, E, \& Mariño, M. (2014). Una comprensión epistemológica de la psicopedagogía. Cinta de moebio, (49), 22-30. [An epistemological understanding of psychopedagogy]. https://dx.doi.org/10.4067/S0717-554X2014000100003

Perochena-González, P, \& Matilde-Coria, G. (2017). La singularidad según la educación personalizada en la era digital. [Uniqueness according to personalized education in the digital age]. Educación, 26(50), 162181. https://dx.doi.org/http://doi.org/1018800/educacion.201701.009 
Rojas-Valladares, A, Estévez-Pichs, M, \& Domínguez-Urdanivia, Y. (2017). Reflexiones acerca de la formación psicopedagógica del estudiante de educación inicial en la universidad metropolitana del Ecuador. [Reflections on the psycho-pedagogical training of the initial education student at the Ecuadorian metropolitan university]. Revista Universidad y Sociedad, 9(4), 44-49. Recuperado de https://url2.cl/hflzw

Romero Oliva, C., \& Montilla-Coronado, M. (2015). La utilización de las TIC en la orientación educativa: un estudio exploratorio sobre la situación actual de uso y formación entre los profesionales de la orientación. [The use of ICT in educational guidance: an exploratory study on the current situation of use and training among guidance professionals]. Revista Española de Orientación y Psicopedagogía, 26 (3), 78-95. Recuperado de https://url2.cl/mclyl

Romero, M., \& Villasmil, J. (2017). Repensar la formación docente. Hacia el encuentro de una nueva perspectiva epistémica para su abordaje y resignificación. [Rethink teacher training. towards the meeting of a new epistemic perspective for its approach and resignification]. CIENCIAMATRIA, 3(5), 133-149. https://doi.org/10.35381/cm.v3i5.17

Sánchez-Cabezas, P., González-Valarezo, M., \& Zumba-Vera, I. (2016). El psicólogo educativo y su responsabilidad en la sociedad ecuatoriana actual: compromisos, retos y desafíos de la educación del siglo XXI. [The educational psychologist and his responsibility in the current Ecuadorian society: commitments, challenges and challenges of the education of the XXI century]. Revista Universidad $y$ Sociedad, 8(4), 123-129. Recuperado de https://url2.cl/YBSu1

Toca-Torres, C., \& Carrillo-Rodríguez, J. (2019). Los entornos de aprendizaje inmersivo y la enseñanza a ciber-generaciones. [Immersive learning environments and teaching cyber-generations]. Educação e Pesquisa, 45 , e187369. https://doi.org/10.1590/s1678-4634201945187369

Vallejo-Valdivieso, P., Zambrano-Pincay, G., Vallejo-Pilligua, P., \& Bravo-Cedeño, G. (2019). Modelos de planificación educativa y diversidad en aulas de clases. [Models of educational planning and diversity in classrooms]. CIENCIAMATRIA, 5(9), 302-315. https://doi.org/10.35381/cm.v6i10.149

Valenzuela, M. (2019). ¿Qué hay de nuevo en la metacognición? Revisión del concepto, sus componentes y términos afines. [What is new in metacognition? Review of the concept, its components and related terms]. Educação e Pesquisa, 45, e187571. https://doi.org/10.1590/s1678-4634201945187571 
Romina Adelina Quevedo-Álava; Luz Adriana Corrales-Moreno; Gema Monserrate Palma-Delgado; Gladis Mercedes Mendoza-Suárez

Vohlonen, A. (2020). COVID-19: Cómo asegurar el aprendizaje de los niños sin acceso a Internet. [COVID-19: How to ensure children's learning without Internet access]. Recuperado de https://url2.cl/RyPdp

Zambrano-Pincay, G., Vallejo-Valdivieso, P., Vallejo-Pilligua, P., \& Bravo-Cedeño, G. (2019). Los profesionales de la Psicopedagogía en la atención a la diversidad como Agente Educativo. [The professionals of the Psychopedagogy in the attention to diversity as an Educational Agent]. Revista Arbitrada Interdisciplinaria de Ciencias de la Salud. Salud y Vida, 3(6), 41-57. http://dx.doi.org/10.35381/s.v.v3i6.304 